\title{
Diagnostic Breast Cancer Image Data Classification using CNN
}

\author{
K. Karthik ${ }^{\text {a }}$, S. Keerthana ${ }^{\text {b }}$ T. K. Dhanyaa ${ }^{c}$ and N. Ramya ${ }^{d}$ \\ Department of Computer Science and Engineering, M.Kumarasamy College of \\ Engineering, Karur, TamilNadu, India- 39113. \\ b,c,d Department of Computer Science And Engineering, M.Kumarasamy College of Engineering, Karur, TamilNadu, India- \\ 639113.
}

Article History: Received: 11 January 2021; Accepted: 27 February 2021; Published online: 5 April 2021

\begin{abstract}
Breast cancer has been dangerous form of cancer. In this report, we use a convolutional neural network to scan and separate infected cells.In this we diagnose if its benign or malignant cancer bulk using computer assisted detection(CAD). The productivity of open $\mathrm{CAD}$ has always been inadequate. Here, we use a deep CNN-based content detection method.We create narrower and broader images of histology patches with cell and tumour attributes. CNN constitutes unorganized data specifically for image data which has been said to be thriving in the area of image recognition. We use highly interconnected layer first cnn, in which those layers are incorporated before the first convolutional layer, since CNN does not support data sets.
\end{abstract}

Keywords: Histology images,CAD,CNN,image detection

\section{Introduction}

Breast cancer is a type of cancer that starts in the breast tissues and spreads throughout the body. It is responsible for a larger number of deaths than other cancers. Breast cancer affects one in every 28 people, according to a study. The breast cancer can occur in two ways benign or malignant. Tumors that won't develop to solid tissue are known as benevolent tumors.Malignant tumours are tumours that can spread to nearby tissues and that can be dangerous.Physical examination requires more amount of understanding and firsthand knowledge of the researchers and also takes a long time. And also the accuracy is less.CAD algorithms have been efficient in disease detection, diagnosis and prediction .

\subsection{Types of Breast Cancer}

Breast cancer can begin in any part of the body. They're divided into groups based on where they came from. a.Ductal cancer is a form of cancer that begins in the duct that transports milk to the nipple.

b.Lobular cancer: Breast cancer develops in the milk-producing glands.A different form of breast cancer is inflammatory breast cancer makes the skin red and feels warm. It formulate the skin appear thick and pitted.

c.Paget disease of the nipple is a cancer that begins in the breast ducts, advances to the nipple tissue, and from there to the dark circles all over the nipple.

d.Phyllodes tumour: This cancer expand in the stroma of the breast

e.Angiosarcoma: The cells that line the lymphatic or blood vessels are where this type of cancer begins.

f.Metastatic breast cancer:The disease has grown across the body. different parts of the body, including the lungs, liver, and other organs.

The CNN is a monitored deep learning consortium of several other stacked convolutional layers that militates between objects. A individual who is fastidious A convolutional layer is used in the CNN inputs, a pooling layer, a recti- fied linear unit (ReLU), batch normalisation, a softmax layer, as well as a completely connected system layer.

Traditionally, digital histology image analyses have largely Traditional classification models like random forests (RF) and support vector machines were ranked first, related to the initial image analysis tasks like nuclei segmentation and feature selection (SVM).Impressive a collection of works zeroed in on the investigation of cores tissue structure and morphology for bosom disease histopathology pictures arrangement.

Kowal et al. [7] tried and thought about 4 distinctive bunching calculations for cores division on 500 bosom minuscule pictures essentially, trailed by extricating 42 morphological, topological and surface highlights utilized in an arrangement strategy with 3 unique classifiers.

George et al. [8] and Filipczuk et al. [9] recognized the areas of the cores with round Houghton change, trailed by bogus favorable disposal utilizing Otsu's sift ageing and different techniques. Subsequent to achieving the division of the cores, dependent on form highlights and sculptural highlights were extricated for old style arrangement prototypes utilized on 92 and 737 bosom clinical cytology pictures individually. Wang et al. [10] zeroed in on the locales in curiosity (ROIs) essentially, at that point split covered cells. Likewise, 4 dependent on form highlights and 138 textural elements highlights dependent on shading there were plenty of empty spaces extricated on 68 pictures for help computer with vectors. Previously mentioned works center around cores division techniques. There are likewise a few works zeroed in on the highlights removed from the entire picture moreover. 
For instance, Naik et al. [11] proposed a procedure that coordinated data from a very low stage data dependent on the pixel esteems, significant degree of difficulty data dependent on connections among pixels, and space explicit data dependent on connections between histopathological designs for location and division of constructions in curiosity. In terms of morphology and atomic highlights were separated for SVM subsequent to utilizing the division calculation. Appropriate data representation is needed for the above-mentioned works to be performed.

A large portion of the effort is devoted to highlight engineering, which is a time-consuming method that extracts valuable features using abundant expert domain knowledge.Furthermore, these studies focused on classifying low-resolution breast cancer histology images into benign or malignant in limited datasets.

pictures Improvements in deep learning and drastic increases in computing capability [12], especially CNN [13], have allowed the progress of computer-assisted analytical approaches to the study of image analysis [14][17], including medical histology.

CNNs learn modules from histology images, as opposed to hand-crafted feature extraction methods. In recent years, an increasing educational institutions have made datasets available that provide multi-class and rising histology images. The exceedingly immense size of a histology picture makes it impossible to teach a CNN.Furthermore, directly rescaling the entire histology image to the CNN input size will result in substantial detail information loss.

As a result, code sampling was used to remove CNN triggering features from sampled patches while still retaining essential information for number of co classification. For their analgesic, Spanhol et al. [18], [19] compiled a dataset of 7909 breast cancer histols. AlexNet was trained using patches extracted haphazardly by a method from breast cancer images with a sliding window and a variety of aspect ratios, and the patch probabilities were combined with three fusion laws for final classification.

To achieve this, our work's key obligations can be summarised as follows: I We recommend a maintenance surveying technique that divides 2 kinds of patches of various widths in needed to shield fundamental data and contain cell- and tissue-level highlights separately (ii) We plan a fix deciding plan to choose more discriminative spots reliant on CNN and K-implies., (iii) We devise a grouping outline that concentrates highlights from patches using element extractors and registers the final element of each entire picture for classification by a classifier.

\section{Literature survey}

[1] In this paper by N.christian et al. there is a proposition to look at the correctnesses of two classifiers to be specific the SVM and Decision Tree (DT) for WBC by utilizing precision pointer to assess order effectiveness of various characterization calculations. By and large, DT characterization precision was discovered to be superior to other classifier in particular the SVM. They could achieve an exactness of $94.54 \%$.In the paper [2], by K.

[2] According to L.E. Boucheron, M. N. Gurcan, et al.2004, factual neural organizations can be used to properly conduct bosom malignant growth determination. On the WBCD data collection, the researcher compared observable neural structure and Multi Layer perception. The Radial Basis Function (RBF), the Probabilistic Neural Network (PNN), and the General Regression Neural Network (GRNN) were used for characterization, and their general introduction was 96.18 percent for RBF, 97 percent for PNN, 98.8 percent for GRNN, and 95.74 percent for MLP. As a result, these observable neural organization constructions have been shown to be useful in analyzing bosom malignancy.

[3] Pluim. J. P. W, Veta.M et al. have done a study on information digging methods for the sake of consistency choice classification. This The most widely used post information digging strategies for the sake of consistency choice and malignancy combining, principally they have zeroed in on the basis of four principles arising a field .They are neural organization based calculations, AI calculations, hereditary calculation and bunch based calculations and they also stated that they will develop in this area in the future.

[4] Spanhol.F.Aet al.2016 has endeavored to execute neural organization for bosom malignant growth finding. Negative connection preparing calculation was utilized to decay an issue consequently and settle them.

In this article, the writer has two methodologies were discussed, for example, transformative methodology and group approach, in which developmental methodology can be utilized to plan thick neural organization consequently. The outfit approach was intended to handle enormous issues yet it was in progress.

[5] B. E. Bejnordi et al. have developed a modernized chest illness examination by uniting innate figuring just as back transmission cranial association that was made as speedier model of classifier to decrease the break down the range of time similarly as building up the accuracy in masterminding a decent number of chest to one of liberal or destructive. In these two cases, distinctive clearing measures was completed on the dataset. In Set A, it just killed records that are lacking data qualities, while set B was prepared with ordinary factual tidying up interaction to perceive any boisterous or omitted qualities. Finally Set A given one hundred percent of most noteworthy exactness rate and Set B received 83.36 percent of the precision. Henceforth the creator possesses achieved this clinical information are best kept in its unique incentive as It provides excellent results exactness rate when contrasted with modified information.

[6] J. Sun and K. He et al.2017 have examined the hereditary calculation and versatile importance hypothesis neural organization for bosom malignancy determination utilizing Data on Breast Cancer in Wisconsin (WBCD). They arranged 699 models which was taken from Fine Needle Aspirates (FNA) with 16 missing data, and 683 models with chest tumors are used in this work of which $65 \%$ was wind up being caring and $35 \%$ furious. 


\section{Methodology}

\subsection{DATASET}

In this segment, set up the dataset utilized in our line of work and pre-preparing in pictures. The data was taken from the bio-imaging 2015 bosom histopathology order test [21], gathered of H\&E and high-resolution (2048 1536 pixels) checked bosom images of malignancy histology.

The digitized pictures with a 200x magnification as well as $0.42 \mathrm{~m}$ pixel scale. Without seeing the territory of interest, two pathologists came up with a name for it the images as odd, kind melanoma in situ or intrusive melanoma, based on the dominant malignant growth type in each picture. The every class's images note inside the database are depicted in Figure1.

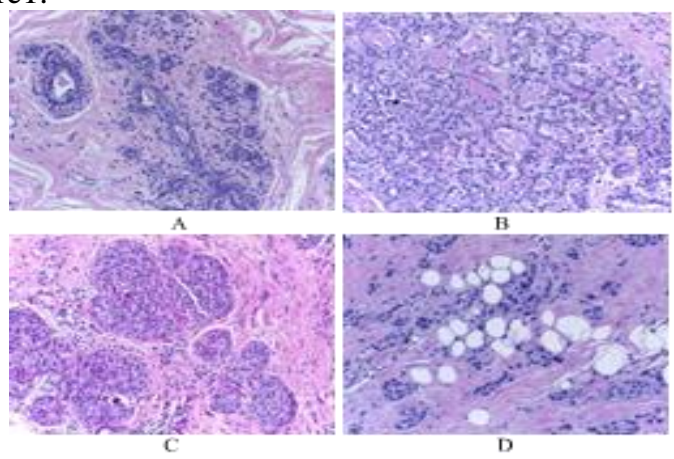

FIGURE 1. H\&E stained images from each type, (A): normal tissue,

(B): benign abnormality, (C): insitu carcinoma, and (D): invasive carcinoma.

\subsection{Preprocess}

Stain inconsistency of histology pictures, because of separation in shading a response of slide advanced scanners, will change the exhibition of picture investigation. As illustrated in Fig. 1, the have in the dataset distinctive blemish variety. In order to do this, stain standardization is essential preceding cycles. There is an assortment of exploration in order to stain standardization the study of histology pictures [26], [27].

In this paper Reinhard et al suggested. [28], which changes the RGB pictures to the decorrelated l $\alpha \beta$ concealing space, then find out the techniques as well as standard deviation each and every direct freely in l $\alpha \beta$ a lot of space of straight conform to arrange the concealing spread of the source and the destination pictures, finally, changes over returning the results to RGB. Fig. 2 shows the method on a chest histology picture.

\subsection{Sampling Patches}

We'll possibly divide the bosom histology image into 4 categories: Classic the skin, kind skin, in squamous cell carcinoma, and invasive carcinoma are the basic forms of cancer. The data removal from the pictures is amazingly subject to an implementation of order. We make use of highlights associated with bosom cells and widespread tissue constructions to relate to every entire picture. Right off the bat, on the grounds that the comprehension of malignant growth cells is amazingly cluttered and the destructive cells have atypicality like better cores and conflicting morphology, therefore, cell-level highlights with the cores data, like shape and fluctuation, just as association of cells highlights like thickness and morphology was used to assess whether or not cells are carcinogenic. The bosom histology images in the dataset have a detection limit of $0.42 \mathrm{~m} 0.42 \mathrm{~m}$, and the cell period is between 3 and 11 pixels. In this manner, we remove little fixes of 128x128pixel resolution to contain highlights at the cell level. In addition, the sick tissue's structure probably irregular.In situ carcinoma is the formation of second-rate carcinogenic or precancerous cells inside a demanding skin layer, such as the testicular pipe, if not causing damage to the surrounding tissue. Obtrusive melanoma, surprisingly, does not exist limit it to the underlying skin container [29]. To distinguish between stage 0 disease and obtrusive tumour, tissue complexes as a whole will be essential in the future.It's unusual for CNNs to simply extract highlights from such a large-scale histology object.

\subsection{Characteristic Extraction}

The histopathologic images display a wide variety of cell morphology, surface, and organisational activities, among other things.For the arrangement job, its display of complicated components is enormous.

The designed in - house include method of separation wants bountiful master space information, and it is work serious and convoluted to separate discriminative highlights.

CNNs can straightforwardly remove agent highlights from pictures, and have accomplished amazing outcomes in different domain.As in the post, ResNet50 [30] is used as a highlight impeller because it is a traditional CNN that is simple to prepare in comparison to other more profound models for the purpose of ensuring the retrieval of quick highlights.

\subsection{Patch Auditing}

This methodology to evaluating patches from histology pictures is depicted in this segment's standard, which is to establish a strategy for monitoring exclusionary $128 \times 128$ pixels spots based on basic AI calculations and ResNet50-128. 


\subsection{Classification Based On Picture}

The grouping of four classes of bosom malignant growth picture dataset, the testing methodology of two sorts of patches, the screening strategy for 128x128 pixels fixes and highlight extractors dependent above has been started on ResNet50. At that point, we modify the take out spots of 512x512 resolution and picked patches of 128 x 128 pixel resolution coordinating to every picture at the preparation batch, and load them into the calibrated ResNet50 and ResNet50-512bunch in a specific order to get the 2048-dimensional highlights gathering, which might reflect the picture's organised phones and tissue frameworks

\section{Result and Discussion}

In our exploration work, we take out more modest patches of $128 \times 128$ pixels and bigger patches of $512 \times 512$ pixels from the mammograms histology pictures in order to have at the cellular and tissue levels highlights, at that point, we examine differentiating $128 \times 128$ pixels patches dependent upon bunching calculation and CNN.

During near tests, it is demonstrated that the two strategies proposed in this paper can proficient final outcome of multi-grouping of bosom histology pictures.

TABLE 1. Comparative results of the recall and accuracy

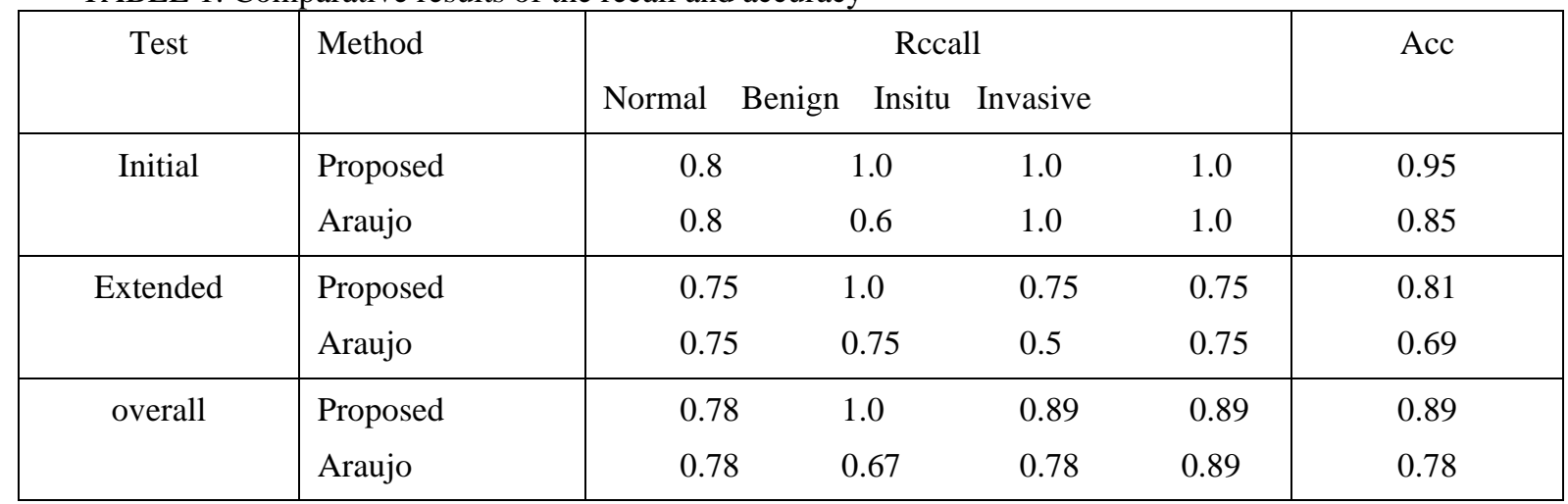

Assess the consequences of the methodology in conjunction with seat tag technique proposed in [20] (CNN SVM) and the similar outcome appears in Table 1. The equivalent dataset extricated patches of 512x512 pixels. CNN planned a finest precision of $77.8 \%$ of many-grouping along with enlarged dataset. It very well may be see that our methodology has a considerable an increase in exactness and review contrasted and the norm strategy, in particular grouping of generous and in situ carcinoma pictures.

To confirm the effect of the screening interaction of $128 \times 128$ pixels patches on the numerous-characterization introduction to bosom malignancy histology pictures, we utilize the ResNet50-128 and ResNet50-512 the element centrifuge in the absence of cycle of calibrating ResNet50-128 along with favored patches that classify of $128 \times 128$ pixels, and afterward, prepare the SVM with every single model patches. Let's take each of the examined patches through the component centrifuge during the testing stage, and then use the P-standard accumulation process to obtain the picture level aspect of each picture for characterization.

Also, Rakhlin et al. [38] being used in excess of a couple of conventional CNNs as highlight centrifuge and inclination helped trees a classification system. Golatkar et al. [39] separated splotches those are wealthy in cores and utilized calibrated Inception-v3.

Those achieve $85 \%$ and $87.2 \%$ precision separately for grouping of four-class utilized $400 \mathrm{H} \& \mathrm{E}$ blemished bosom histology pictures in the total dataset everywhere for Breast Cancer Histology Challenge (BACH). In comparison to other cutting-edge methods, our approach, for example, is extreme.

TABLE 2:The performance of multi-classification based on patches of $512 \times 512$ pixels

\begin{tabular}{|c|c|c|c|c|}
\hline Result & Normal & Benign & In situ & Invasive \\
\hline Precision Recall & 0.78 & 0.78 & 0.7 & 1.0 \\
& 0.78 & 0.78 & 0.78 & 0.89 \\
\hline F-Score & 0.780 & 0.780 & 0.738 & 0.942 \\
\hline
\end{tabular}

The disarray grids of the exploration are uncovered in Table 2. The picture shrewd precision of the general test bunch is $80.56 \%$. The exactness, review and F1-score of every class are appeared on Table 1. As indicated by the recipe (5), the determined consequence of full scale $\mathrm{F}$ is $81.0 \%$.

\section{Conclusion}

In the article, we actualize the successful strategy to arrange the H\&E blemished bosom histology pictures into 4 classes: typical tissue, favorable injury, stage 0 disease and invasive tumour. Because of the side effects of destructive cells and the variety morphology of the tissues and constructions among in situ melanoma and 
intrusive melanoma, we take out 2 sorts of patches of 512x512 pixels and 128x128 pixels from the histology pictures to have exceptional stages highlights.

\section{References}

1. Yang.Y“An evaluation of statistical approaches to text categorization,’' Inf. Retr., vol. 1, nos. 1-2, pp. 6990, 1999.

2. Reinhard.E M. Adhikhmin, P. Shirley, and B. Gooch, "Color transfer between images,' IEEE Comput. Graph. Appl., vol. 21, no. 5, pp. 34-41, Sep./Oct. 2001.

3. Tabar.LVitak.B H. H. Chen, M. F. Yen, S. W. Duffy, and R. A. Smith, "Beyond randomized controlled trials: organized mammographic screening substantially reduces breast carcinoma mortality," Cancer, vol. 91, no. 9, pp. 1724-31, 2001.

4. Murugesan, M., Thilagamani, S. ," Efficient anomaly detection in surveillance videos based on multi layer perception recurrent neural network", Journal of Microprocessors and Microsystems, Volume 79, Issue November 2020, https://doi.org/10.1016/j.micpro.2020.103303

5. S. W. Duffy, L. Tabar, H. H. Chen, M. Holmqvist, M. F. Yen, S. Abdsalah, B. Epstein, E. Frodis, E. Ljungberg, C. Hedborg-Melander, A. Sundbom, M. Tholin, M. Wiege, A. Akerlund, H. M. Wu, T. S. Tung, Y. ---H. Chiu, C. P. Chiu, C. C. Huang, R. A. Smith, M. Rosen, M. Stenbeck, and Holmberg.L "The impact of organized mammogra- phy service screening on breast carcinoma mortality in seven swedish counties," Cancer, vol. 95, no. 3, pp. 458-69, 2002.

6. Kopans.D.B "Beyond randomized controlled trials: organized mammographic screening substantially reduces breast carcinoma mortality," Cancer, vol. 94, no. 2, pp. 580-1; author reply 581-3, 2002.

7. Naik.S S. Doyle, A. Madabhushi, M. Feldman, J. Tomaszewski, and S. Agner, "Automated gland and nuclei segmentation for grading of prostate and breast cancer histopathology,' in Proc. 5th IEEE Int. Symp., May 2008, pp. 284-287.

8. Thilagamani, S., Nandhakumar, C. ." Implementing green revolution for organic plant forming using KNNclassification technique”, International Journal of Advanced Science and Technology, Volume 29 , Isuue 7S, pp. 1707-1712

9. Deng.JDong.W L.-J. Li, K. Li, L. Fei-Fei, and Socher.R "ImageNet: A large-scale hierarchical image database," in Proc. IEEE Conf. Comput. Vis. Pattern Recognit., Jun. 2009, pp. 248-255.

10. M. Macenko et al., 'A method for normalizing histology slides for quantitative analysis,' in Proc. IEEE Int. Symp. Biomed.Imag., Nano Macro (ISBI), Jun./Jul. 2009, pp. 1107-1110.

11. L. He, L. R. Long, S. Antani, and G. Thoma, 'Computer assisted diagnosis in histolopathology,' Sequence Genome Anal., Methods Appl., vol. 3, $\quad$ pp. 271-287, 2010.

12. Thilagamani, S., Shanti, N.," Gaussian and gabor filter approach for object segmentation", Journal of Computing and Information Science in Engineering, 2014, 14(2), 021006, https://doi.org/10.1115/1.4026458

13. L. He, L. R. Long, S. Antani, and G. R. Thoma, "Histology image analysis for carcinoma detection and grading," Comput Methods Programs Biomed., vol. 107, no. 3, pp. 538-556, 2012.

14. Krizhevsky.A.,Sutskever, I., Hinton, G., 2012. Imagenet classification with deep convolutional neural networks. In: Advances in Neural Information Processing Systems. pp. 1097-1105

15. Krizhevsky.A I. Sutskever, and G. E. Hinton, “ImageNet classification with deep convolutional neural networks,' in Proc. 25th Int. Conf. Neural Inf. Process. Syst., Lake Tahoe, NV, USA, Dec. 2012, pp. 10971105.

16. Rhagini, A., Thilagamani, S. ,'Women defence system for detecting interpersonal crimes",International Journal of Advanced Science and Technology, 2020, Volume 29,Issue7S, pp. 1669-1675

17. Bengio, Y., Courville, A., Vincent, P., 2013. Representation learning: A review and new perspectives. IEEE Trans Pattern Anal Mach Intell 35 (8), 1798-1828

18. Filipczuk.P T. Fevens, A. Krzyzak, and R. Monczak, "Computer-aided breast cancer diagnosis based on the analysis of cytological images of fine needle biopsies,'’ IEEE Trans. Med. Imag., vol. 32, no. 12, pp. 2169 2178, Dec. 2013

19. Veta.M J. P. W. Pluim, M. A. Viergever, and P. J. van Diest, “Breast cancer histopathology image analysis: A review," IEEE Trans. Biomed. Eng., vol.61, no. 5, pp. 1400-1411, May2014.

20. K.Deepa, S.Thilagamani, "Segmentation Techniques for Overlapped Latent Fingerprint Matching", International Journal of Innovative Technology and Exploring Engineering (IJITEE), ISSN: 2278-3075, Volume-8 Issue-12, October 2019. DOI: 10.35940/ijitee.L2863.1081219. 
21. Girshick.R J. Donahue, T. Darrell, and J. Malik, "Rich feature hierarchies for accurate object detection and semantic segmentation," in Proc. IEEE Conf. Comput. Vis. Pattern Recognit. (CVPR), Jun. 2014, pp. 580587.

22. Y. M. George, H. H. Zayed, M. I. Roushdy, and B. M. Elbagoury, "Remote computer-aided breast cancer detection and diagnosis system based on cytological images," IEEE Syst. J., vol. 8, no. 3, pp. 949-964, Sep. 2014.

23. Russakovsky. O, Deng, J., Su, H., Krause, J., Satheesh, S., Ma, S., Huang, Z., Karpathy, A., Khosla, A., Bernstein, M., Berg, A. C., FeiFei, L., 2014. ImageNet large scale visual recognition challenge.Int J Comput Vis $115(3), 1-42$.

24. Y. LeCun, Y. Bengio, and G. Hinton, "Deep learning," Nature, vol. 521, pp.436-444,May2015. ]Makki.J "Diversity of breast carcinoma: Histological subtypes and clin- ical relevance," Clin. Med. Insights, Pathol., vol. 8, pp. 23-31, Dec. 2015. [26]J. Gu et al. (2015). "Recent advance in convolutional neural networks."'[Online]. Available: https://arxiv.org/abs/1512.07108

25. Santhi, P., Mahalakshmi, G., Classification of magnetic resonance images using eight directions gray level co-occurrence matrix (8dglcm) based feature extraction, International Journal of Engineering and Advanced Technology, 2019, 8(4), pp. 839-846.

26. Pêgo.A,Aguiar.P(2015).Bioimaging.[Online].Available:http://www.bioimaging2015.ineb.up.pt/dataset.html

27. S. Vahadane et al., "Structure-preserved color normalization for histological images," in Proc. IEEE 12th Int. Symp. Biomed.Imag.(ISBI),Apr.2015,pp.1012-1015

28. Y. LeCun, Y. Bengio, and G. Hinton, “Deep learning,” Nature, vol. 521, no.7553,pp.436-444, 2015.

29. D.B Kopans, "An open letter to panels that are deciding guidelines for breast cancer screening," Breast Cancer Res Treat, vol. 151, no.1, pp.19-25, 2015.

R. L. Siegel, K. D. Miller and Jemal.A "Cancer statistics, 2015,"

30. He.Kand Sun.J "Convolutional neural networks at constrained time cost," in Proc. IEEE Conf. Comput. Vis. Pattern Recognit., Jun. 2015, pp. 5353-5360.

L. Siegel, K. D. Miller andJemal.A “Cancer statistics, 2015," CA cancer journal for clinicians, vol. 65, no. 1, pp. 5-29, 2015.

31. Santhi, P., Lavanya, S., Prediction of diabetes using neural networks, International Journal of Advanced Science and Technology, 2020, 29(7 Special Issue), pp. 1160-1168.

32. Spanhol.F.A L. S. Oliveira, L. Heutte, and C. Petitjean, "A dataset for breast cancer histopathological image classification,” IEEE Trans. Biomed. Eng., vol. 63, no. 7, pp. 1455-1462, Jul. 2016.

33. Vijayakumar, P, Pandiaraja, P, Balamurugan, B \& Karuppiah, M 2019, 'A Novel Performance enhancing Task Scheduling Algorithm for Cloud based E-Health Environment', International Journal of E-Health and Medical Communications (IJEHMC), Vol 10,Issue 2,pp 102-117.

34. K. He, X. Zhang, J. Sun, and S. Ren, "'Deep residual learning for image recognition," Vis. Pattern Recognit., 2016, pp. 770-778.

35. Wang.P X. Hu, Q. Liu, X. Zhu, and Y. Li, "Automatic cell nuclei segmentation and classification of breast cancer histopathology images,” Signal sProcess., vol. 122, pp. 1-13, May 2016.

36. Pandiaraja, P, Vijayakumar, P, Vijayakumar, V \& Seshadhri, R 2017, 'Computation Efficient Attribute Based Broadcast Group Key Management for Secure Document Access in Public Cloud', Journal of Information Science and Engineering, 33, No. 3, pp. 695-712.

37. Wang.D, A. Khosla, R. Gargeya, H. Irshad, and A. H. Beck. (2016). "Deep learning for identifying metastatic breast cancer.'[Online]. Available: https://arxiv.org/abs/1606.05718

38. N Deepa , P. Pandiaraja, 2020 ," Hybrid Context Aware Recommendation System for E-Health Care by merkle hash tree from cloud using evolutionary algorithm", Journal of Soft Computing, Springer, Volume 24 ,Issue 10, Pages 7149-7161.

39. X. Zhu, J. Yao, J. Huang, and F. Zhu, "WSISA: Making survival prediction from whole slide histopathological images," in Proc. IEEE Conf. Comput. Vis. Pattern Recognit., Jul. 2017, pp. 7234-7242.

40. N Deepa , P. Pandiaraja, 2020, "E health care data privacy preserving efficient file retrieval from the cloud service provider using attribute based file encryption “, Journal of Ambient Intelligence and Humanized Computing, Springer, https://doi.org/10.1007/s12652-020-01911-5.

41. B. E. Bejnordi "Context-aware stacked convolutional neural net- works for classification of breast carcinomas in whole-slide histopathology images," J. Med. Imag., vol. 4, no. 4, p. 044504, 2017.

42. T. Araújo et al., "Classification of breast cancer histology images using convolutional neural network," PLoS ONE, vol. 12, no. 6, p. e0177544, 2017 
43. P. Pandiaraja, N Deepa 2019 ," A Novel Data Privacy-Preserving Protocol for Multi-data Users by using genetic algorithm”, Journal of Soft Computing, Springer, Volume 23 ,Issue 18, Pages 8539-8553

44. Mary.A.K(2018).BreasTCancer.[Online].Available:https://www.merckmanuals.com/home/women-shealth-issues/breast-disorders/ breast-cancer

45. K Sumathi, P Pandiaraja 2019," Dynamic alternate buffer switching and congestion control in wireless multimedia sensor networks", Journal of Peer-to-Peer Networking and Applications, Springer, Volume 13,Issue 6,Pages 2001-2010..

46. Golatkar, D. Anand, and Sethi.A "Classification of breast cancer histology using deep learning," in Proc. Int. Conf. Image Anal. Recognit. Cham, Switzerland: Springer, 2018, pp. 837-844. 